\title{
Pneumatosis Intestinalis and Intestinal Perforation in a Patient Receiving Tocilizumab
}

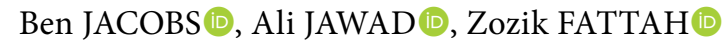 \\ Department of Rheumatology, Barts Health NHS Trust, London, United Kingdom
}

\begin{abstract}
In this article, we report a case of pneumatosis intestinalis associated with tocilizumab use. This is a unique case and may explain the increased rate of idiopathic bowel perforation among patients taking tocilizumab.

Keywords: Intestinal perforation; pneumatosis intestinalis; tocilizumab.
\end{abstract}

The presence of gas within the wall of the small or large intestine is referred to as pneumatosis intestinalis (PI). The pathogenesis is poorly understood. It has been described with gastrointestinal or non-gastrointestinal conditions. In a small number of cases, it was idiopathic. ${ }^{1}$ Despite being a benign condition in most cases, life-threatening complications may occur. ${ }^{1}$ In this article, we describe a patient who developed PI and intestinal perforation while taking tocilizumab.

\section{CASE REPORT}

A 68-year-old male patient was noted to have pneumoperitoneum with sub-diaphragmatic gas and a Rigler sign (Figure 1) on routine surveillance chest radiograph. For the last year, he was on weekly subcutaneous tocilizumab $162 \mathrm{mg}$. The patient was admitted for emergency imaging to exclude a bowel perforation. Contrast-enhanced computed tomography of the abdomen showed widespread intramural bowel gas throughout the colon without obvious perforation (Figure 2). There was a suggestion of small bowel stricture. As there was no clinical evidence of peritonitis or perforation, the patient was discharged from hospital. Tocilizumab therapy was withheld due to the theoretical risk of bowel perforation. A written informed consent was obtained from the patient.

Ten years previously, he presented with symmetrical polyarthritis. Serology revealed a positive rheumatoid factor and citrullinated C-peptide, antinuclear and anti U1-ribonucleoprotein (RNP) antibodies. His complement levels were normal and antibodies against cardiolipin, Ro, Sm, Jo-1 and double stranded deoxyribonucleic acid were negative. A diagnosis of rheumatoid arthritis (RA) was established.

He was started on weekly oral methotrexate (10 mg). Three months later, golimumab $50 \mathrm{mg}$ monthly subcutaneous injections were added

Received: October 12, 2017 Accepted: November 03, 2017 Published online: January 17, 2018

Correspondence: Ali Jawad, MD. Department of Rheumatology, Barts Health NHS Trust, E1 4DG London, United Kingdom. Tel: 00442082238408 e-mail: alismjawad1@hotmail.com 


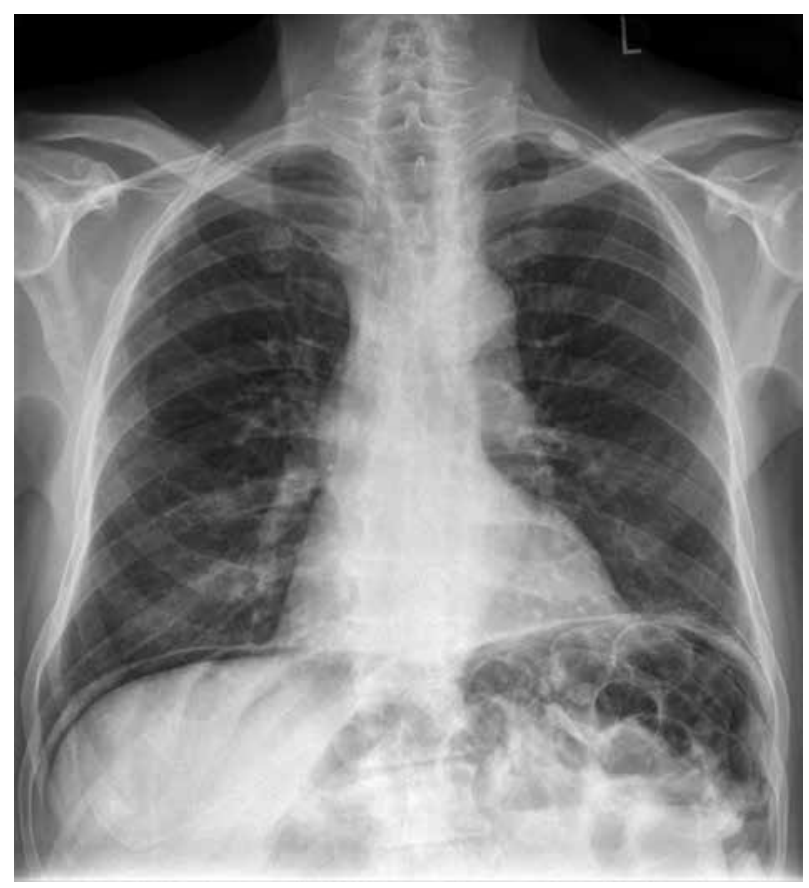

Figure 1. Erect chest radiograph demonstrating pneumoperitoneum.

with a good response. Fifteen months later, he developed interstitial lung disease. Golimumab and methotrexate were stopped and he was started on leflunomide $20 \mathrm{mg}$ daily. His antibody profile did not change. Fifteen months later, he developed livedo reticularis, digital vasculitis, and peripheral neuropathy. This was treated with prednisolone and withdrawal of the leflunomide. The prednisolone was gradually tapered off and tocilizumab was started. His arthritis and the vasculitic process responded to therapy.

Three months after admission, repeat chest radiographs demonstrated resolution of the pneumoperitoneum prior to starting hyperbaric oxygen therapy. Nonetheless, the patient underwent two courses of hyperbaric oxygen therapy.

A follow-up small bowel magnetic resonance imaging study demonstrated a probable $20 \mathrm{~cm}$ stricture of the terminal ileum. Diagnostic laparoscopy failed to show any abnormality. Colonoscopy showed two sessile polyps in the ascending and transverse colon which were removed. The terminal ileum could not be entered.

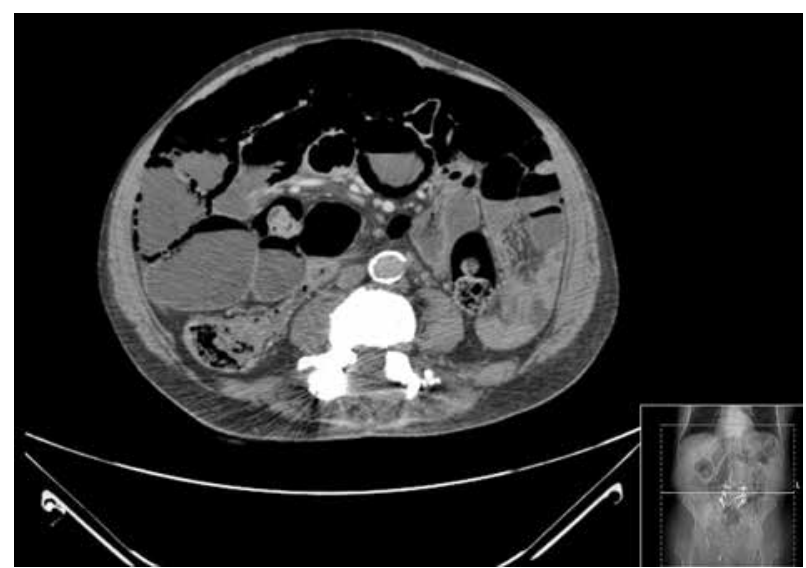

Figure 2. Abdominal computed tomography scan demonstrating pneumoperitoneum and pneumatosis intestinalis.

\section{DISCUSSION}

Tocilizumab is a recombinant human interleukin-6 (IL-6) immunoglobulin G1 monoclonal antibody that competes for both membrane-bound and soluble forms of IL- 6 receptors, decreasing signal transduction through gp130. ${ }^{1}$ IL- 6 exerts pleiotropic effects on innate and adaptive immunity including orchestrating the acute phase response, neutrophil recruitment and $\mathrm{T}$ cell differentiation. ${ }^{2}$ IL-6 signaling is an important part of the proinflammatory cytokine milieu which initiates and perpetuates inflammation in RA. ${ }^{3}$ Therapeutic targeting of the IL- 6 pathway with tocilizumab is now an evidence-based option for treating selected patients with RA. 4-8 Well-documented adverse effects of tocilizumab include anaphylaxis, severe or atypical infections, gastrointestinal perforation, malignancy, bone marrow suppression, and derangement of liver enzymes.

Pneumatosis intestinalis is a radiologic sign defined by the presence of gas within the intestinal wall. ${ }^{9-11} \mathrm{PI}$ often presents as an incidental finding on abdominal imaging in asymptomatic patients, but it may occur in the context of life-threatening intestinal pathology, such as acute intestinal ischemia.11 Many patients present with vague, usually mild abdominal symptoms such as diarrhea, hematochezia, abdominal pain, constipation, weight loss, and tenesmus. ${ }^{11}$ It is difficult to determine whether symptoms are due to PI itself or to the underlying cause, if an underlying cause is present. 
In $15 \%$ of cases, no specific underlying etiology has been identified.111 Recognized secondary causes of PI include ischemic colitis, necrotizing enterocolitis (in infants), and instrumentation of the gastrointestinal tract, infectious colitis, immunocompromised states, chronic lung disease, and immunosuppressive drugs such as glucocorticoids. ${ }^{11}$ It has also been associated in rare cases with primary amyloidosis, autoimmune connective tissue, and vascular diseases. ${ }^{10}$

The pathogenesis of PI is poorly understood. The mechanical theory proposes gas tracks from the intestinal lumen via the mucosa or from the lungs via the mediastinum entering the intestinal wall. The bacterial theory proposes that gas-forming organisms such as Clostridium perfringens breach the mucosa and subsequently generate gas-filled pseudocysts. These theories are not mutually exclusive.

The natural history of pneumoperitoneum associated with PI is also poorly understood..$^{10}$ Various reports in the literature described cases of PI-associated pneumoperitoneum which led to unnecessary abdominal surgery, with no evidence of perforation visible at operation. ${ }^{11-14}$

Tocilizumab is associated with an unexplained increased rate of bowel perforation. ${ }^{15}$ The British Society for Rheumatology guidelines recommend using tocilizumab with caution in patients with a history of diverticular disease, and suggest that the concomitant use of non-steroidal antiinflammatory drugs or glucocorticoids increases the risk further. ${ }^{5}$ The rate of perforation is approximately 2.7 per 1,000 patient-years, as compared to 0.2 to 0.6 per 1,000 patient-years for patients with RA receiving other disease modifying anti-rheumatic drugs. Interestingly, the majority of patients who develop bowel perforation on tocilizumab do not have preexisting diverticular disease, possibly because of the uncertainty regarding the increased risk of perforated diverticula. Many of these patients presented with mild, non-specific symptoms rather than with an acute abdomen. This finding may be due to masking of the systemic inflammatory response by tocilizumab or due to a benign underlying etiology. ${ }^{16}$

We propose that in this case, tocilizumab predisposed to the development of PI. Subsequently, rupture of an intramural gas-filled pseudocyst led to pneumoperitoneum in the absence of frank intestinal perforation.

The observation that withdrawal of tocilizumab was followed by rapid resolution of the pneumoperitoneum adds weight to our hypothesis. It was also possible that PI was secondary to the suspected ileal stricture, and that tocilizumab merely promoted the development of pneumoperitoneum while dampening the accompanying inflammatory response that one would expect. The significance of the positive anti U1-RNP antibodies in our patient is not clear.

Despite the lack of a definitive explanation for this patient's condition, we believe that this case highlights a novel potential complication of tocilizumab therapy. To our knowledge, there are no published case reports in the literature linking tocilizumab and PI. This case demonstrates that tocilizumab may be associated with the development of PI and subsequent pneumoperitoneum. This clinical picture may be alarming if the clinician is unfamiliar with the condition, and may lead to unnecessary investigation and management, including unnecessary emergency laparotomy.

Further studies are required to elucidate the relationship between tocilizumab and bowel perforation. It will be of great interest to document the findings of PI in cases of tocilizumabassociated bowel perforation, as PI may explain this mysterious and worrying adverse effect.

\section{Declaration of conflicting interests}

The authors declared no conflicts of interest with respect to the authorship and/or publication of this article.

\section{Funding}

The authors received no financial support for the research and/or authorship of this article.

\section{REFERENCES}

1. Heng Y, Schuffler MD, Haggitt RC, Rohrmann CA. Pneumatosis intestinalis: a review. Am J Gastroenterol 1995;90:1747-58.

2. Hunter CA, Jones SA. IL- 6 as a keystone cytokine in health and disease. Nat Immunol. 2015;16:448-57.

3. McInnes IB, Schett G. Cytokines in the pathogenesis of rheumatoid arthritis. Nat Rev Immunol 2007;7:429-42. 
4. Singh JA, Beg S, Lopez-Olivo MA. Tocilizumab for rheumatoid arthritis. Cochrane Database Syst Rev 2010;7:CD008331.

5. Malaviya AP, Ledingham J, Bloxham J, Bosworth A, Buch M, Choy E, et al. The 2013 BSR and BHPR guideline for the use of intravenous tocilizumab in the treatment of adult patients with rheumatoid arthritis. Rheumatology (Oxford) 2014;53:1344-6.

6. Smolen JS, Beaulieu A, Rubbert-Roth A, RamosRemus C, Rovensky J, Alecock E, et al. Effect of interleukin- 6 receptor inhibition with tocilizumab in patients with rheumatoid arthritis (OPTION study): a double-blind, placebo-controlled, randomised trial. Lancet 2008;371:987-97.

7. Maini RN, Taylor PC, Szechinski J, Pavelka K, Bröll J, Balint G, et al. Double-blind randomized controlled clinical trial of the interleukin- 6 receptor antagonist, tocilizumab, in European patients with rheumatoid arthritis who had an incomplete response to methotrexate. Arthritis Rheum 2006;54:2817-29.

8. Strand V, Burmester GR, Ogale S, Devenport J, John A, Emery P. Improvements in health-related quality of life after treatment with tocilizumab in patients with rheumatoid arthritis refractory to tumour necrosis factor inhibitors: results from the 24-week randomized controlled RADIATE study. Rheumatology (Oxford) 2012;51:1860-9.

9. Ho LM, Paulson EK, Thompson WM. Pneumatosis intestinalis in the adult: benign to life-threatening causes. AJR Am J Roentgenol 2007;188:1604-13.

10. Pear BL. Pneumatosis intestinalis: a review. Radiology 1998;207:13-9.

11. St Peter SD, Abbas MA, Kelly KA. The spectrum of pneumatosis intestinalis. Arch Surg 2003;138:68-75.

12. Daly BD, Guthrie JA, Couse NF. Pneumoperitoneum without peritonitis. Postgrad Med J 1991;67:9991003.

13. Schulenburg A, Herold C, Eisenhuber E, Oberhuber G, Volc-Platzer B, Greinix HT, et al. Pneumatosis [correction of Pneumocystis] cystoides intestinalis with pneumoperitoneum and pneumoretroperitoneum in a patient with extensive chronic graft-versus-host disease. Bone Marrow Transplant 1999;24:331-3.

14. Imai K, Doi Y, Takata N, Yoshinaka I, Harada K. Successful conservative treatment of pneumatosis intestinalis associated with intraperitoneal free air: report of a case. Surg Today 2012;42:992-6.

15. Strangfeld A, Richter A, Siegmund B, Herzer P, Rockwitz K, Demary W, et al. Risk for lower intestinal perforations in patients with rheumatoid arthritis treated with tocilizumab in comparison to treatment with other biologic or conventional synthetic DMARDs. Ann Rheum Dis 2017;76:504-10.

16. Conway R, Orr C, McCarthy GM. Lesson of the month 1: Septic arthritis with normal acute phase reactants and white cell count in a patient receiving tocilizumab. Clin Med (Lond) 2017;17:280-1. 